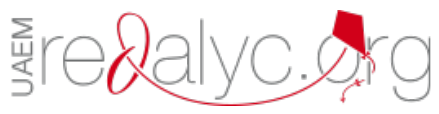

Centro Sur

ISSN: $2600-5743$

compasacademico@icloud.com

Grupo Compás

Ecuador

\title{
La adicción y sus diferentes conceptos
}

Trujillo Segrera, Mónica Andrea

La adicción y sus diferentes conceptos

Centro Sur, vol. 3, núm. 2, 2019

Grupo Compás, Ecuador

Disponible en: http://www.redalyc.org/articulo.oa?id=588861691002

Esta obra está bajo una Licencia Creative Commons Atribución-NoComercial-SinDerivar 4.0 Internacional. 


\title{
La adicción y sus diferentes conceptos
}

\author{
Addiction and its different concepts \\ Mónica Andrea Trujillo Segrera mtrujillo@uniminuto.edu \\ Corporación Universitaria Minuto de Dios, Colombia \\ http://orcid.org/0000-0002-2393-1380
}

Centro Sur, vol. 3, núm. 2, 2019

Grupo Compás, Ecuador

Recepción: 18 Enero 2017 Aprobación: 24 Julio 2018

Redalyc: http://www.redalyc.org/ articulo.oa?id $=588861691002$
Resumen: En este artículo se realizará una revisión temática sobre el concepto de adicciones; La cual inicia desde el concepto de "adiciones" como tal, citado para el Ministerio de sanidad y consumo de España, haciendo un recorrido desde los años 60, década en la cual la Organización Mundial de la salud OMS hace referencia a "Dependencia" termino que se da después de una discusión entre "adicción" y "habituación” entre los años 1920 y 1960. Así mismo en el CIE-10, para el alcoholismo o la adicción a las drogas se implementa la terminología "trastornos mentales y del comportamiento debido al consumo de sustancias psicotrópicas”, en el área de psicopatologías se evidencia una clasificación en dos grupos "dependencia física" y "dependencia psíquica” (Beloch A, Sandin B, Ramos F, 2008). Por otra parte, el DSM V habla de otros dos grupos de adicciones los "Trastornos relacionados con sustancias y trastornos adictivos" y los "Trastornos no relacionados con sustancias", como es el caso del juego patológico codificado (F63.0), (Asociación Americana de Psiquiatría, 2013, p.316). y finalmente se resalta la adicción como una enfermedad que debe ser atendida por las entidades y profesionales de la salud, como los dicta el Congreso de Colombia en la ley 1566 del 31 de julio del 2012, en las cuales nombran las normas para garantizar la atención integral a personas que consumen sustancias psicoactivas.

Palabras clave: Adicciones, dependencia, drogadicción, enfermedad.

Abstract: In this article there will be a thematic review on the concept of addictions; Which starts from the concept of "additions" as such, cited for the Ministry of health and consumption of Spain, making a journey since the 60s, decade in which the WHO World Health Organization refers to "Dependence" term that occurs after a discussion between "addiction" and "habituation" between the years 1920 and 1960 . Likewise in the ICD-10, for alcoholism or drug addiction the terminology "mental and behavioral disorders due to the consumption of psychotropic substances ", in the area of psychopathologies there is evidence of a classification into two groups" physical dependence "and" psychic dependence "(Beloch A, Sandin B, Ramos F, 2008). On the other hand, the DSM V talks about two other groups of addictions the "Disorders related to addictive substances and disorders" and the "Disorders not related to substances", as is the case of the codified pathological game (F63.0), (Association American Psychiatry, 2013, p.316). and finally addiction is highlighted as a disease that must be attended by health entities and professionals, as dictated by the Colombian Congress in law 1566 of July 31,2012 , in which they appoint the rules to guarantee care comprehensive to people who consume psychoactive substances.

Keywords: Addictions, dependency, drug addiction, disease.

\section{INTRODUCCIÓN}

Frente a la inmersión en el tema de adicciones desde el área de psicología específicamente orientada desde la academia y la investigación, se identificó la variedad de términos frente al concepto de adicciones, de esta manera se hace necesario generar un escrito que oriente a los estudiantes interesados en el tema y a otros que los introduzca y sensibilice 
frente a este fenómeno tan importante para el conocimiento de los profesionales de la salud.

El tema de adicciones es un concepto amplio, ya que involucra el individuo, familia y comunidad, con fuertes influencias sociales, económicas, políticas, jurídicas y tecnológicas, así mismo desde el individuo se aborda el área física y psicológica. Por tal motivo es un término que involucra diversidad de conceptos desde diferentes campos de conocimiento.

Desde el área de la salud en el Glosario de términos de alcohol y drogas del ministerio de sanidad y consumo de España (1994) se define a la "Adicción, a las drogas o alcohol" como "Consumo repetido de una o varias sustancias psicoactivas, hasta el punto de que el consumidor (denominado adicto) se intoxica periódicamente o de forma continua, muestra un deseo compulsivo de consumir la sustancia (o las sustancias) preferida, tiene una enorme dificultad para interrumpir voluntariamente o modificar el consumo de la sustancia y se muestra decidido a obtener sustancias psicoactivas por cualquier medio".

El concepto de "adicción" es polemizado y en ocasiones usado con terminología diferente, las cuales son definidas desde cada una de las perspectivas de los autores en representación a diferentes áreas de conocimiento interesadas en el tema.

De esta manera desde el área de la salud entre las décadas de 1920 y 1960, se debatió el término de "adicción" y "habituación" siendo en los años 60, remplazado por "dependencia” por la Organización Mundial de la salud, OMS. Tanto así que el término "adicción” no figura en la "Clasificación internacional de enfermedades" CIE10, pero aun así es empleado por muchos profesionales de la salud. (Ministerio de Sanidad y consumo de España,1994)

\section{MATERIALES Y MÉTODOS}

En el CIE-10, para el alcoholismo o la adicción a las drogas se implementa la terminología "trastornos mentales y del comportamiento debido al consumo de sustancias psicotrópicas (f10-f19)" en donde hace referencia al "uso, abuso y dependencia de sustancias psicoactivas" (CIE 10, 1996). De esta manera la categoría representa la sustancia psicoactiva, es decir F10 para trastornos relacionados con alcohol y F11 para los trastornos relacionados con opiáceos, y el cuarto carácter indica el patrón de consumo, 1 para abuso, 2 para Dependencia y 9 para consumo. En este caso se puede decir el alcohol se referenciaría de la siguiente manera. F10.1 Abuso de alcohol, F10.2 Dependencia de alcohol, y F10.9 Consumo de alcohol no especificado. (CIE 10, 1996) En este sentido es necesario conocer las características de cada uno de los patrones de consumo. 


\begin{tabular}{|l|l|}
\hline CIE 10 & OTROS AUTORES \\
\hline $\begin{array}{l}\text { Consumo: no se detectan } \\
\text { sobre el concias inmediatas } \\
\text { Su entorno }\end{array}$ & $\begin{array}{l}\text { Uso: Dosis moderadas; } \\
\text { Habito: Consumo más } \\
\text { frecuente, No aumentar la } \\
\text { dosis, ni se padecen } \\
\text { trastornos físicos o } \\
\text { psicológicos importantes, su } \\
\text { búsqueda no deriva en } \\
\text { alteraciones conductuales. } \\
\text { (Graña, Muñoz y Navas, 2007) }\end{array}$ \\
\hline $\begin{array}{l}\text { Abuso: se producen } \\
\text { ensecuencias negativas para consumidor y lo su entorno. }\end{array}$ & $\begin{array}{l}\text { Abuso: consumo en Exceso; } \\
\text { presentan "consecuencias a } \\
\text { nivel social, biológico y } \\
\text { psicológico (Pérez F, 2011) }\end{array}$ \\
\hline $\begin{array}{l}\text { Dependencia: Integra la } \\
\text { dimensión física y psíquica. }\end{array}$ & $\begin{array}{l}\text { Dependencia: Se puede hablar } \\
\text { de dependencia psiquica, } \\
\text { dependencia física y } \\
\text { dependencia social. (Pérez F, } \\
\text { 2011) }\end{array}$ \\
\hline
\end{tabular}

Los autores

En el campo de la salud, principalmente en Psiquiatría y Psicología es necesario para los profesionales relacionados con el campo de las adicciones, conocer las diferentes características de los patrones de consumo.

\section{RESULTADOS}

Uso: Dosis moderadas; en donde se presenta "utilización, en momentos puntuales, con la intención de probar tan sólo o el capricho de atesorar una experiencia esporádica”. (Perez F, p.211). En relación se habla también de Habito: Como un "consumo más frecuente; No existe una tendencia a aumentar la dosis, ni se padecen trastornos físicos o psicológicos importantes cuando la sustancia se consigue, por lo que su búsqueda suele ser limitada y nunca deriva en alteraciones conductuales"

Abuso: En El Real Colegio de Psiquiatras Británico se formuló en 1987 la siguiente definición: "se entiende por abuso cualquier consumo de sustancias químicas que dañe o amenace con dañar la salud física, mental o el bienestar social de un individuo, de diversos individuos o de la sociedad en general". Así mismo se puede abusar de un objeto como es el caso de la ludopatía. Según el CIE 10, "el abuso" es entendido como un "consumo perjudicial” en el que se encuentra el abuso social, abuso ocasional. Según el DSM V, para diagnosticarse abuso, la persona, debe haber abusado continuamente durante doce meses o bien debe ser persistente.

Dependencia: La dependencia Psicológica, está vinculada a la necesidad de corte emocional en el caso de las drogas se habla de "craving" como deseo irreprimible de repetir la conducta consumatoria (Perez F, 2011). La dependencia física es la necesidad del organismo de una sustancia, la dependencia social, es la necesidad de consumir alguna sustancia o deseo de pertenecer a algún grupo social, como son las personas que son dependientes de otras también llamadas, co-dependientes o 
bi-dependientes, los cuales presentan el trastorno de "personalidad dependiente" ya que creen que sin el otro no pueden vivir. (Perez F, 2011). Por lo general estos no actúan como elementos separados, sino complementarios e interactuantes en un mismo sujeto.

Desde las perspectivas Psicopatológica, se plantean diferencias entre la "dependencia física" y la "dependencia psicológica" o comúnmente conocidas como "adicciones físicas" y "adicciones psicológicas".

La "dependencia física" hace referencia a "estado de adaptación que se manifiesta por la aparición de intensos trastornos físicos cuando se interrumpe la administración de la droga o influye en su acción por la administración de un antagonista especifico. Esos trastornos, esto es, los síndromes de abstinencia, están construidos por series específicas de síntomas y signos de carácter psíquico y físico peculiares de cada tipo de droga"

Mientras que la "dependencia Psíquica" se define como aquella "situación en la que existe un sentimiento de satisfacción y un impulso psíquico que exigen la administración regular o continua de la droga para producir placer o evitar malestar" (p.15).

Dando continuidad a las diferentes terminologías relacionadas con el concepto de "adicción" es importante reconocer otro término como lo es la "drogodependencia" la cual hace referencia a la "dependencia motivada por una sustancia”. (Perez F, 2011). El término Drogodependencia ha tenido una constante evolución, inicialmente se hacía referencia a "Viciosos" que atribuía a la falta de voluntad, posterior a este en el siglo XIX se hablaba de "toxicómano" teniendo como el sufijo "manía" el cual tiene connotaciones morales. El uso del término "drogadicción" tiende a ser limitante en algunos casos, donde se presentan dependencias diferentes a las drogas, por lo cual es más frecuente el empleo del término "adicción" por diferentes profesionales, teniendo en cuenta que el término "dependencia" también es usado a nivel médico, refiriéndose a otras sustancias que no son perjudiciales para la salud, sino por el contrario complementan alguna deficiencia en el cuerpo, es decir son perentorios, como lo cita Alonso-Fernández "en medicina la dependencia física se refiere al hecho de que una persona necesite una sustancia, digamos la insulina".

\section{CONCLUSIONES}

Si bien el término "adicción" es comúnmente utilizado, es importante tener claro que desde el Manual Diagnóstico y Estadístico de los Trastornos Mentales de la Asociación Americana de Psiquiatría DSM $\mathrm{V}$, no hace referencia al concepto de "adicción" como diagnóstico, sino que lo enuncia como "Trastornos relacionados con sustancias y trastornos adictivos" en sus diferentes clases de sustancias: alcohol, cafeína, cannabis, alucinógenos, inhalantes, opiáceos, estimulantes, tabaco, otras sustancias o sustancias desconocidas; recientemente se incluyen los "Trastornos no relacionados con sustancias", como es el caso del juego patológico codificado (F63.0). A partir de esta vinculación, en cuanto a la ludopatía 
como trastorno mental, es claro que se puede depender de algo que no sea una "sustancia", por ejemplo, de un objeto, la OMS en su artículo de junio del 2018 publica la nueva clasificación de las enfermedades del (CIE-11) en el cual El trastorno del videojuego se ha añadido a la sección relativa a trastornos de adicción. De esta manera se puede hablar de dos grandes grupos de adicciones, las adicciones químicas y las adicciones comportamentales.

Es importante reconocer las drogas sin duda alguna afectan al cerebro, "enfermedad del cerebro porque las drogas cambian al cerebro: modifican su estructura y cómo funciona".

Aunque se han propuesto distintos tipos de adicciones, hoy podemos diferenciarlas en dos grandes grupos: aquellas producidas por sustancias químicas y aquellas producidas por conductas. Serían las adicciones químicas y las adicciones comportamentales.

Por este motivo es importante que los profesionales de la salud, enfermeras, médicos, psiquiatras, psicólogos entre otros, sean conscientes que la adicción es una enfermedad y como cualquier otra, debe ser tratada, la persona que lo padezca debe ser atendida de manera oportuna.

De esta manera El Congreso de Colombia en la ley 1566 del 31 de julio del 2012, dictan las normas para garantizar la atención integral a personas que consumen sustancias psicoactivas. En el artículo $1^{\text {o "Reconózcase que }}$ el consumo, abuso y adicción a sustancias psicoactivas, lícitas o ilícitas es un asunto de salud pública y bienestar de la familia, la comunidad y los individuos. Por lo tanto, el abuso y la adicción deberán ser tratados como una enfermedad que requiere atención integral por parte del Estado, conforme a la normatividad vigente y las Políticas Públicas Nacionales en Salud Mental y para la Reducción del Consumo de Sustancias Psicoactivas y su Impacto, adoptadas por el Ministerio de Salud y Protección Social”.

En el momento que los profesionales se apropien de esta normatividad e identifiquen a la adicción como una enfermedad, siendo conscientes del impacto a nivel individual y social, estaremos hablando de un gran aporte a este fenómeno, ya que a partir de esto las familias y la comunidad vinculada a estas, podrán analizar a las adicciones desde otra perspectiva, no desde un punto de vista criminal, sino como un persona enferma, que necesita atención integral desde el área de la salud y otras que apoyen el proceso.

\section{Referencias}

De Leon, G. (2009). La comunidad terapéutica y las adicciones teoría, modelo y método. España: Editorial Desclée de Brouwer.

Ministerio de sanidad y consumo de España. (1994). Glosario de términos de alcohol y drogas, España. Recuperado de http://apps.who.int/iris/ bitstream/handle/10665/44000/9241544686_spa.pdf? sequence $=1$ \&isAllowed $=\mathrm{y} \& u a=1$

OMS. (2018). La Organización Mundial de la Salud (OMS) publica hoy su nueva Clasificación Internacional de Enfermedades (CIE-11). Recuperado 
de http://www.who.int/es/news-room/detail/17-06-2018-who-releasesnew-international-classification-of-diseases-(icd-11)

Nora D. Volkow, M.D., (2008). Las drogas, el cerebro y el comportamiento: Las drogas, el cerebro y el comportamiento: La ciencia de la adicción, Instituto Nacional sobre el Abuso de Drogas (NIDA). Recuperado de https:// d14rmgtrwzf5a.cloudfront.net/sites/default/files/soa_sp.pdf

Pérez del Rio, F. (2011). Estudios sobre adicciones. España: EXCMA

El congreso de Colombia. (2012). Ley 1566. Colombia. Recuperado de https:// docs.supersalud.gov.co/PortalWeb/Juridica/Leyes/L1566012.pdf

Beloch, A., Sandin, B., Ramos, F., (2008). Manual de Psicopatología. España: McGrawHill. 